\title{
DISCLAIMER
}

\begin{abstract}
This report was propared as an acoount of work apeneored by an asency of the Unitod Statea Government. Neither the United States Gove'nment nor any aseacy thereof, nor any of their employees, makes any werranty, expreas or impliod, or asumes alyy logal liability or responaibility for the accuracy, completenows, or usefulnewe of any information, apparatun, product, or procees dierloed, or represente that its use would not infringe privalely owned rights. Refor. ence heroin to any apecific commercial product, proceas, or cervioe by trede name, tredemart, manufucturer, or otherwise doen not neccesearlily conetitute or imply its endorsement, recom. mendation, of favoring by the United States Oovernment on any agancy thereof. The viowe and opinions of suthon exprumed herein do not mecomarily state or reflect those of the United States Gowernment or any apency thereof.
\end{abstract}

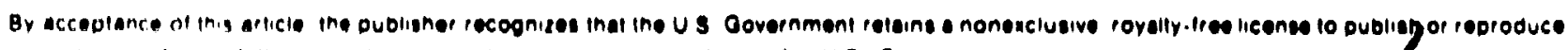

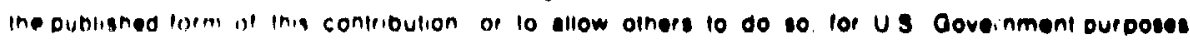


Theoretical Division, Los Alamos National Laboratory Los Alamos, NM 87545 U.S.A.

and

W. B. Kaufmann

Department of Physics, Arizona State University

Tempe, AZ 85287 U.S.A.

\begin{abstract}
Recent pion absorption daca with the observation of two protons In colncidenca have been interpreted to yleld a small fraction of absorption on two-nucleon palra compared with absorption on multinucleon clusters. The present work demonstrates that a cunsiderable broadening of the two-nucleon correlation function, and concurrent reduction of observed events, arises fiom Fermi motion, nucleon binding, pion ecattering before absorption and nucleon scattering after absorption. The width of the calculated pp angular correlation distribution agraes well with that observed experimentally. After correction for these effects in the assumed two-body absorption process we find that there is a residual mechanlsm which must account for -30 of the true-absorption events.
\end{abstract}

\title{
I. Introduction
}

The mechentsm of plon absorption in nuclel has been studied for several years. Racent interest has cencered around the number of nucleons involved In the abeorption process. 2 , To atudy this issue two experimente have recontly been performed 2,3 in the resonance region in which two protone are observed in colncldence following plon absorption. A distinct peak is seen in the correlation function at the angle between the two protons corresponding to absorption on free-nucleon palr ("free kinematlc value"). By Integrating the meashred cross sections ovar all angles, a total "two-nucleon" absorption crosn section 1s ojtalned. The authors of reference 1 conclude that only about 20 of plon absorptions occur on a palr of nucleons, a eurpelelngly smail nuraber.

In extracting this probability, however, they must declde how much of the angular diatribution around tho free klinematic value ahould be Included a part of the two-nucloon, a distinct from a multi-nucleon, absorption mechenian. Since the shape of the curve 1s not Gausslar., but has "winge", they declded to remove a 
"background" from the curve. The subtraction is made from $\mathrm{d} \sigma / \mathrm{d} \Omega$ and assunes symmetry about the Eree kinematics point. The solid-angle factor makes the background correction substantial (factors of 2 to 5) and not just a few percent, as it might appear on their plot.

The "quasideuteron model" assumes that pions annihilate on pn pairs having quantum numbers of the deutergn, $i . e ., I-0, L-0$, and $\mathrm{S}=1$. It was pointed out by Ritchie et $a 1 .{ }^{4}$, that, if one takes this model seriously then $\pi$-absorption data on ${ }^{12} \mathrm{C}$ may be understood qualitatively (in PWIA) without the need of a multinucleon absorption mechanism. The ieuteron mav be in a state with angular momentum either zero or two relative to the ${ }^{10} \mathrm{~B}$ core. The $\ell-0$ component gives a peak similar to the central peak observed, while the $\ell-2$ component gives a broader distribution resembling the wings and having a minimum at the two-body kinematic point. The calculation is difficult to extend to heavier nuclei because the spectroscopic factors for $l=0$ and $l-2$ (or higher) deuterons are not known. Also the inelastic pion and nucleon scattering, not treated rigorously in Ref. 4, become more important as A increases.

The piesent talk takes a different approach, that of an intranuclear cascade (INC) model. In its present form the technique does not give detailed attention to the shell structure; it is more appropriate fģr heavier nuclel. In this article we analyze recent data on ${ }^{68} \mathrm{Ni}$. In contrast to the work of ref. 4 the INC method has the advantage of Including "distortions" due to the inelastic scattering of plons and the scattering of the outgoing nucleons.

We should note that there are a large number of technlques used to calculate nuclear reactions. The methods vary according to the degree of "exclusivity" Involved. For elastic scattering, or transitions leading to a definite final state, a totally quancum. mechanical description must be used since phase linformation is very important. For reactions in which there are thousands of firal states it is assumed tha: the phase information is largely lost and semi-classlcal alculations may represent the process well. Table I gives the spectium of calculations on the left, matched with processes on the $r$ ight. Of course unitarlty gives us relations among these quantities and there is the famous "end-run", which is to use the optical theorem on the elastlc amplitude on one ent to obtain the total cross section on the other.

\section{Calculational Techndaue}

We first ketch the fntranuclear cascade model, which is more fully described elsowhere ${ }^{5}$.

We assume all nucleons move clasaically in a Woods-Saxon potontial. Each particle is chosen to have a definite total energy, depending on $1 \mathrm{ts}$ shell. For the case treated here, the ohells and their binding energles are shown in Table II. The nucleons are initlally cast in a uniform spheriol distribution with the 
Optical model, DWIA, coupled ch.annels

Sum of DWIA or Fermi gas model DWIA to single density

Sum of DWIAS

INr+Pauli Blockingtthree body absorption

INC+Pauli Blocking

Intra-Nuclear Cascade
Elastic scattering, inelastic scatt. to a single state, pion absorption to a given final state Reactions to several final states Giant resonances, single particle states

Reaction to final states in several nuclei

Inclusive absorption on 3 nucleons

Inclusive absorption on two nucleons

Total cross section

Classical Meshanics

Table I. Spectrum of calculational techniques matched to the reaction types.

She 11

$$
\text { Number of }
$$
Nucleons in Shell

$\ell$

$\begin{array}{lrll}\text { Is } & 4 & 0 & 40 \\ \text { Ip } & 12 & 1 & 30 \\ \text { Id } & 20 & 2 & 25 \\ 2 s & 4 & 0 & 10 \\ \text { If } & 18 & 3 & 10\end{array}$

Binding Energy ( $\mathrm{MeV}$ )

Table II. Standard binding energles used in the present calculation.

magnitude of thelr momenta dictated by their binding energies and their potential energy within the well. The initlal directions of these momente are chosen 1sotropically. In one version thr nucleons In a shell with angular momentum $l$ are selected so as to have the angular momeata in the range $(l, l+1)$. Thls procedure simulates the quantum mochanical shell structrure. The angular momentum of each nucleon is conserved by its equation of motion in the absence of collistons with other nucleons. Wh1le the model nucleus has, on average, zero angular momentum, it is made up of nucloons with fint te $l$, as in nature. There will, of course, also be a dlatrlbution of palrs with angular momenta, usually non-zoro, glven by the classical rules outlined above, so that the effect of ref, 4 is Included, but in a classical manner. Neutrons and protons are distinguished only in a satistical sense. 
The mean field described in the previous paragraph represents the average of the long-range fart of the nucleon-nucleon interaction. The short-range part of the force is represented by nucleon-nucleon collisions, which occur if the nucleons approach each other within a distance corresponding to a cross section of $\sigma_{N N}$, a parameter in the model. The nucleons prupagate relativistically and the $\mathrm{N}-\mathrm{N}$ collisions are isotropic in the $\mathrm{NN}$ center-of-mass frame.

The pion, treated with relativistic kinematics, collides with nucleons with a probability governed by the pion-nucleon total cross section at the appropriate laboratory energy. The angular distribuition of the scattering is taken from a representation of experimental da=a.

No absorption is allowed on the first pion-nucleon collision. In subsequent collisions absorption takes place with a fixed probability $P$, another parameter in the model. The energy and momentum of the absnrbed pion are shared by the current and previous nucleon. The additional canceling nucleon momenta, corresponding to the pion mass, are chosen along the direction of the pion motion between the two nucleons. If $\mathrm{P}-1$ (and two collisions occur) then the pion will be absorbed on the first palr it encounters.

\section{II . Extraction of Observables}

The analysis is performed by selacting all pairs of nucleons which have the sum of their kinetic energies greater than some fixed value $E_{c}$. If the momentum of the incident pion is $\bar{k}$ and the two nucleon momenta are $\bar{p}_{1}$ and $\dot{p}_{2}$ then the angle, $\theta$, between $\dot{p}_{2}$ and $\bar{k}-\dot{p}_{1}$ measures the deviation from absorption on a free nucleon pair. The effects of refraction of the outgoing protons, Farmi motion, nucleon-nucleon collisions and Interaction of the pion with nucleons before absorption will lead ro a dispersion in this angle. The first two effects are treated under the general title of Fermi motion.

There are four principal sources of redurtion of the twonucleon corralation functions relative to absorption as free nucleon palrs.

1. Eermi mction A large fraction of the two-nucleon ahsorption iakes place when clie angle of one of the nucleons in the center of mass is about $30^{\circ}$. The backward moving nucleon, in motion concrary to the center-of-mass movement of the composite system, has a relatively low kinetic energy, of the order of $65 \mathrm{MeV}$. Because of the Ferml motion of the pair thls energy is spread. Consequently some fraction of the lower-energy members of the palr fall below the cut-of $F$ energy of the counters ( $25 \mathrm{MeV}$ In thls case), and hence the rucieon pair is not observed.

2. Bundure Eneray While the bindlig energy of the last nucloon is moderate ( $10 \mathrm{MeV}$ in this case) cho binding of the interlor nucitoon is much grcater. Since even "two-nucleon" 
interior nucleons is much greater. Since even "two-nucleon" absorption is a multinucleon process (in the sense that the pion typically hits several nucleons during the absorption event) it is not predominantly a surface process. Thus the interior nucleons may be expected to play a large role in pion absorption. In the present model the number of nucleons participating in absorption in a given shell is proportional to the number of nucleons in that shell, independent of binding energy, i.e., no appreciable shadowing is observed. The binding energy of each shell is well known from knock-out reactions. For a pion absorbed on a pair of nucleons in the 1 s shell the loss is substantial. Approximately 208 of the twonucleon absorptions are lost to observation from the Fermi motion and binding alone.

3. Nucleon Scattering After Absorption Either nucleon (or both) may be scattered before leaving the nucleus. There has been some debate about the importance of this effect because of the long mean-free-path ( $m f p$ ) inferred from optical potentials derived from proton scattering. This long $m f p$ is presumed to be due to the Pauli blocking of the struck nucleon.

For this reason we discuss in some detall our method for calculating the nucleon collisions. The present calculatior divides the nucleon nucleon interaction into two parts. The first part is long range and makes up the attractive well which binds the nucleus. The effect of this potential has already been included since it is tha source of the binding energy in (2) above. There remains a strong short-rance interaction which leads to the high-energy scattering cross section of $-40 \mathrm{mb}$. Thus the cross section used in these calculations is only a fraction of the total cross section for low energy nuclear collisions. The mfp in nuclear matter corresponding to this value is about $1.7 \mathrm{fm}$. The effect of Pauli blocking is taken into account by ignoring any collision which would lead to a final state in which elther nucleon would have an energy such that it would be bound by more than a fixed energy $E$ (the "Ferml level", typlcally $10 \mathrm{MeV}$ in our calculations). ThPs lengthens the mfp considerabiy. We have ascertalned, by recording the histories of the two nucleons recelving the absorption energy, that about one half of the collisions are Paull blocked. Thus the actual mfp in nuclear matter would be $-3.5 \mathrm{fm}$. Of course many of the reactions are not in the center of the nucleus so the effective density is smaller and the actual "mfp" is longer. Despite the strong aependence of the mfp on Paull blocking of the NN collision:i, this effect plays a rather small role in the reduction in the number of correlated proton palrs seen. If the Paull blocking were turned off the only collisions affected would be those in which the outgolng nucleon transfers a small amount of energy to the bound one. The result would be sllght broadening of the correlation function and not a loss in the number of pp palrs observed. Fer the optical model any nucloon-nucloon elastic scattering, no matter how soft, would lead to a loss of flux if allowed to occur. The fact that the small-energy-loss (small-angle) Inelastlc scatterlngs are the ones suppressed may be seen directly in the angular distribution 
discuss the relationship of corrections to the two-nucleon correlation function in terms of the nucleon $\mathrm{mfp}$ without specifying more details of the nucleon scattering.

4. Pion-Nucleon Collisions The pion must make at least two collisions before it can be absorbed on the last two nucleons with which it collided. The probability per collision is $P$ in the calculation. This value is chosen to reproduce the observed twonucleon cross section. The pion may collide with $0,1,2, \ldots$ nucleons before being ausorbed on the final pair. Thus it may lose a sufficient fraction of its kinetic energy so that the event is not counted in the two-nucleon correlation measurement. This effect was discussed by Girija and Koltun.

\section{Results}

The present intranuclear cascade code includes all of the effects described in the previous section. We can study each separately by means of parameter changes. The results are summarized in Table III for pion absorption on ${ }^{5}{ }^{3} \mathrm{Ni}$ with an incident pion laboratory momentum of $270 \mathrm{MeV} / \mathrm{c}$. The losses are composed of several small effects, all acting in concert until only about 20 s of the events remain.

These results are exprossed in terms of a reduction factor defined by $R-\sigma\left(E_{c}, E_{0}\right) / \sigma$ where $\sigma\left(E_{c}, E_{0}\right)$ (is the inclusive $\left(\pi, N_{1} N_{2}\right)$ cross section summed over all $\mathrm{N}_{1} \mathrm{~N}_{2}$ pairs. The cross sections are

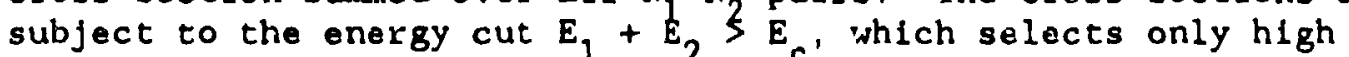
energy pairs, and the energy cuts $E_{1} \mathcal{F}^{\prime} E_{0}$ and $E_{2}>E_{0}$, which correspond to the low-energy cutoff of the detectors. $\sigma$ is the total pion absorption cross section. For comparison with ref. 3 we have chosen $E_{c}-160,230 \mathrm{MeV}$ and $E_{0}-25 \mathrm{MeV}$.

$R$ is commonly less than one because Inelastic scattering of the incident pion or rescatsering of the nucleons on which the absorption takes place lower the energy of the observed nucleon nalr. It is also possible that one member of the nucleon pair is left in a bound state and so is not observed. Because the sum extends over all nucleon pairs, the absorption results in one very fast and one slow nucleon. The slower nucleon may strike another, knocking it out of the nucleus. Provided $E$ is not too great, elther of the two slow nucleons may be palred with the fast one to satisfy $E_{1}+E_{2}>E_{c}$. Some cf this "overcounting" occurs even if $E_{c}$ - $230 \mathrm{MeV}$. The redúction factor is not very sensitive to the detalled parameters of the nucieon collision process and, as can be seen from Table III, actually lncreases slightly when the nucleon collision cross section is increased.

In Table III the flrst run (row 1) corresponds to a "PWIA" calculation. Since $P$ is unity tine plon must annihilate on the first two nucleons it encounters. There is no "Initial state Interaction". The condftion ${ }^{\circ} \mathrm{NN}^{-0}$ means that the outgoing nucleons Interact only with the potential well. There are no secondary knock-out processes. The rediction factor comes complately fron the Fermi motion and binding energy corrections as discussed in III.I and III. 2 . 
Fermi motion and binding energy corrections as discussed in III.1 and III. 2 .

In the calculation of row 2, $P$ is reduced to 0.4 . This allows scattering of the incident $\pi^{+}$before its annihilation. The $\pi^{+}$ shares its rinetic energy among several nucleons during the "prescatters" so there is a depletion of the high energy $(230 \mathrm{MeV})$ events. The lowe: energy events increase because of there now may be several pp pairs.

A large reduction in (especially high energy) events due to the final NN scatters in seen in row 3 . Rows 4 and 5 include both $\pi$ prescattering and NN scattering.

The relative insensitivity to Pauli blocking mentioned in III. 3 may be seen by comparing 5, 6, and 7 . The $E_{B}-10 \mathrm{MeV}$ case corresponds to full blocking of the highest shell, while the $E_{B}=$ $60 \mathrm{MeV}$ case corresponds to no blocking. The case in which $\Sigma E>230$ $\mathrm{MeV}$ is hardly affected. The case for which $\Sigma E>160 \mathrm{MeV}$ is more affected because "soft" scatters of the outgoing proton may enhance the overcounting pieviously discussed.

A comparison of rows 6 and 8 show the lack of sensitivity to small changes is $\sigma_{\mathrm{yN}}$. Rows 5 and 9 show insensitivity to the precise value of $P^{N N}$. Runs 9 and 10 differ only in that the angular momentum selection is not imposed on run 10; the direction of the momentum is chosen randomiy for each nucleon.

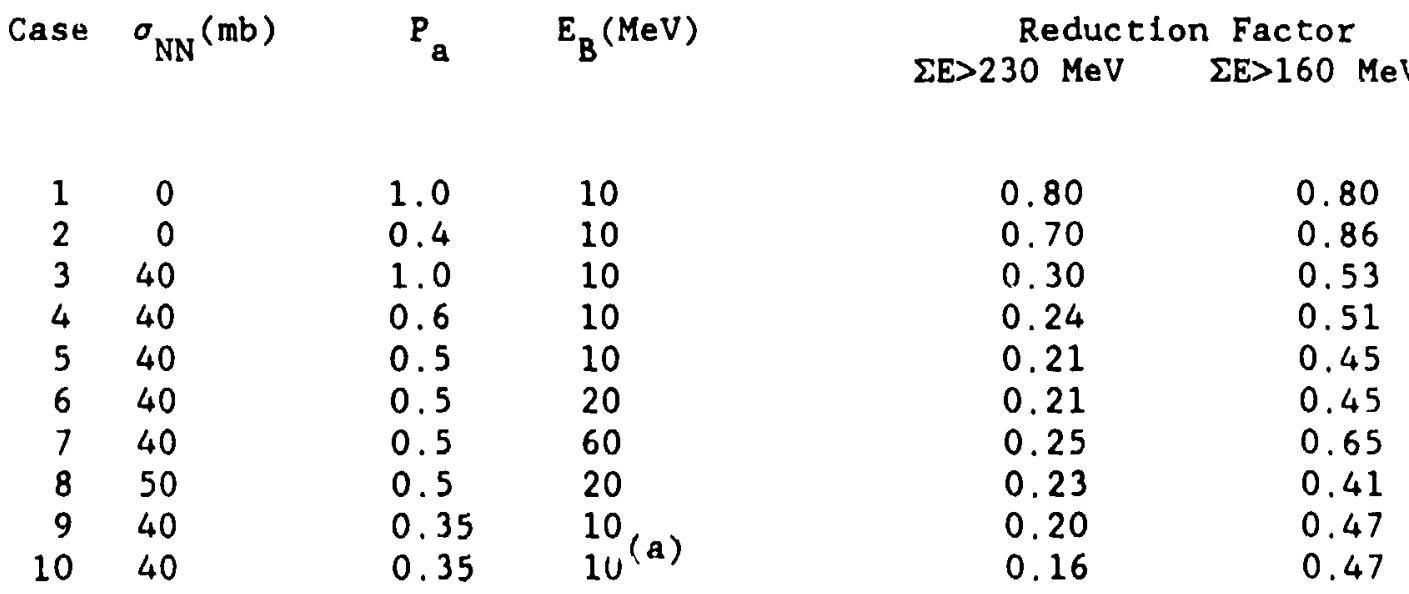

Table III. Dependence of the reduction factor on the various mechanisms in the calculation. The label "a" indicates that the individual shells were not required to have a specific angular momentum corresponding to that shell. Different shapes result for the individual shell densities.

We note that ref. 3 measured only the p-p palrs following annihllation so that $i t$ is necessary to correct for the n-p pairs, which we will assume have the same reduction factors. We note, from ref. 1, that the high energy pfoton spectrum, near the quas1-twobody kinematical point, from $\pi$ projectiles (leading to pp palrs) is 
nine times larger than the corresponding proton point from $\pi$ projectiles (leading to np pairs). Taking into account that there are two protons for each $\pi^{+}$event and only one for each $\pi^{*}$ event the np pairs are 228 of the pp pairs. Since ${ }^{58} \mathrm{Ni}$ has nonzero isospin, a correction (the number of np pairs over the number of pp pairs) must be applied which leads to a factor to obtain all two nucleon events of 1.25 , i.e., the cross section observed in ref. 3 should be increased 258, as should the inferred two nucleon absorption cross section.

Yet another possible correction arises from the fact that the counters used in ref. 3 had a loss of energy discrimation for protons with energy greater than $200 \mathrm{MeV}$. This involves about 128 of the pairs with summed energy greater than $230 \mathrm{MeV}$. However only about 28 would be lost to the cross section, the other 108 still having enough energy (after counting all energies oreater than 200 $\mathrm{MeV}$ to be only $200 \mathrm{MeV}$ ) to satisfy the energy criterion at $230 \mathrm{MeV}$. We do not include this correction in the present work.

The results are summarized in Table IV. If one now uses values of the fotal true-absorptfon cross section as measured by Ashery et al. ${ }^{1}$, or Nakai et al. ${ }^{2}$, a residual absorption cross section can be inferred. A substantial fraction remains but we see that the errors cause the effect to be marginal in statistical terms. We have not included, as errors, the uncertainty due to the calculation. We show instead two variations corresponding to angular momentum selection (which correspond in turn to changes in shapes of the individual shell densities).

\begin{tabular}{|c|c|c|c|}
\hline $\begin{array}{l}\text { Two proton } \sigma \\
(\mathrm{mb})\end{array}$ & $\begin{array}{l}\text { Two nucleon } \sigma \\
(\mathrm{mb})\end{array}$ & $\begin{array}{l}\text { Residual Absorptid } \\
\text { Ashery ( } 577 \pm 90 \mathrm{mb}) \\
\quad(\mathrm{mb})\end{array}$ & $\begin{array}{l}\text { Cross Section } \\
\text { Nakai ( } 527 \pm 109 \mathrm{mb}) \\
(\mathrm{mb})\end{array}$ \\
\hline $\begin{array}{l}285 \pm 20 \\
356 \pm 25\end{array}$ & $\begin{array}{l}357 \pm 25 \\
446 \pm 31\end{array}$ & $\begin{array}{ll}220 \pm 93 & (38 \pm 16) \\
131 \pm 95 & (23 \pm 16)\end{array}$ & $\begin{aligned} 170 \pm 108 & (32 \pm 20) \\
81 \pm 109 & (15 \pm 21)\end{aligned}$ \\
\hline
\end{tabular}

Table IV. Results of the analysis.

The figure shows the angular correlation functions for ${ }^{58} \mathrm{~N} 1$ for two different energy selection conditions chosen for comparison with ref. 3. While the contributions from the nucleon collision and pion collision effects change with the cut on energy, the Fermi motion broading does not. Note that, due to the solid angle factor, the larger angles are very important.

The distinction between two-rucleon absorption and multi. nucleon absorption in such a classical calculation is somewhat arbitrary. All of the results presented here are in the two-nucleon category in the sense that when the pion is absorbed all of the energy corresponding to its rest mass is given to two nucleons only. 
Certainly the case of Fermi motion alone corresponds to a pure twonucleon absorption mechanism. The authors of ref. 2 do not consider the addition of final nucleon collisions to be multi-nucleon absorption but rather two-nucleon absorption with final state scattering. For the pion interactions before the absorption the same might be said.

We gratefully acknowledge several helpful discussions with B. Ritchie and R. Reciwine.

This work was supported by the U. S. Department of Energy and the Associated Western Universities.

\section{REFERENCES}

1. R. D. Mckeown et al., Phys. Rev. Lett. 44, 1033 (1980);

D. Ashery, et al., Phys. Rev. C23, 2173 (1981); V. Girija and

D. S. Koltun, BAPS 28, 746 (1983).

2. A. Altman, et al., Phys. Rev. Lett., 50, 1187 (1983).

3. W. J. Burger, Jr., et al., Phys. Rev. Lett. 57, 58 (1986).

4. B. G. Ritchie, N. S. Chant and P. G. Roos, Phys. Rev . C30, 969 (1984); D. Ashery, Phys. Rev. C32, 333 (1985); B. G. Ritchie et al., Phys. Rev. C32, 334 (1985).

5. D. Strottman and W. R. Gibbs, Phys. Lett. 149B, 288 (1984);

W. R. Gibbs and D. Strottman, Proceedings of the International Conference on Antinucleon- and Nucleon-Nucleus Interactions, Telluride, March 1985

6. Handbook of Pfon-Nucleon Scattering, G. Höhler, F. Kaiser, R. Koch and E. Pletarinen, Fachinformationzentrum, ISSN 0344-8401, Karlsruhe (1979).

7. G. Jacob and Th. A. J. Maris, Rev. Mod. Phys., 38, 121 (1966), 45, 6 (1976); A. E. L. Diperink and T. deForest, Ann. Rev. Nuc1. Sc1. 25, 1 (1975).

8. J. Schiffer, Nuc1. Phys. 335,348 (1980).

9. J. $\because$. Negele, Comments Nuc1. Part. Phys. 12, 1 (1983).

10. V. Girifa and D. S. Koltun, Phys. Rev. C31, 2147 (1985).

11. D. Ashery tt a1., Phys. Rev. C23, 2173 (1981).

12. K. Nukal, et al., Phys. Rev. Lett. 44, 1446 (1980). 

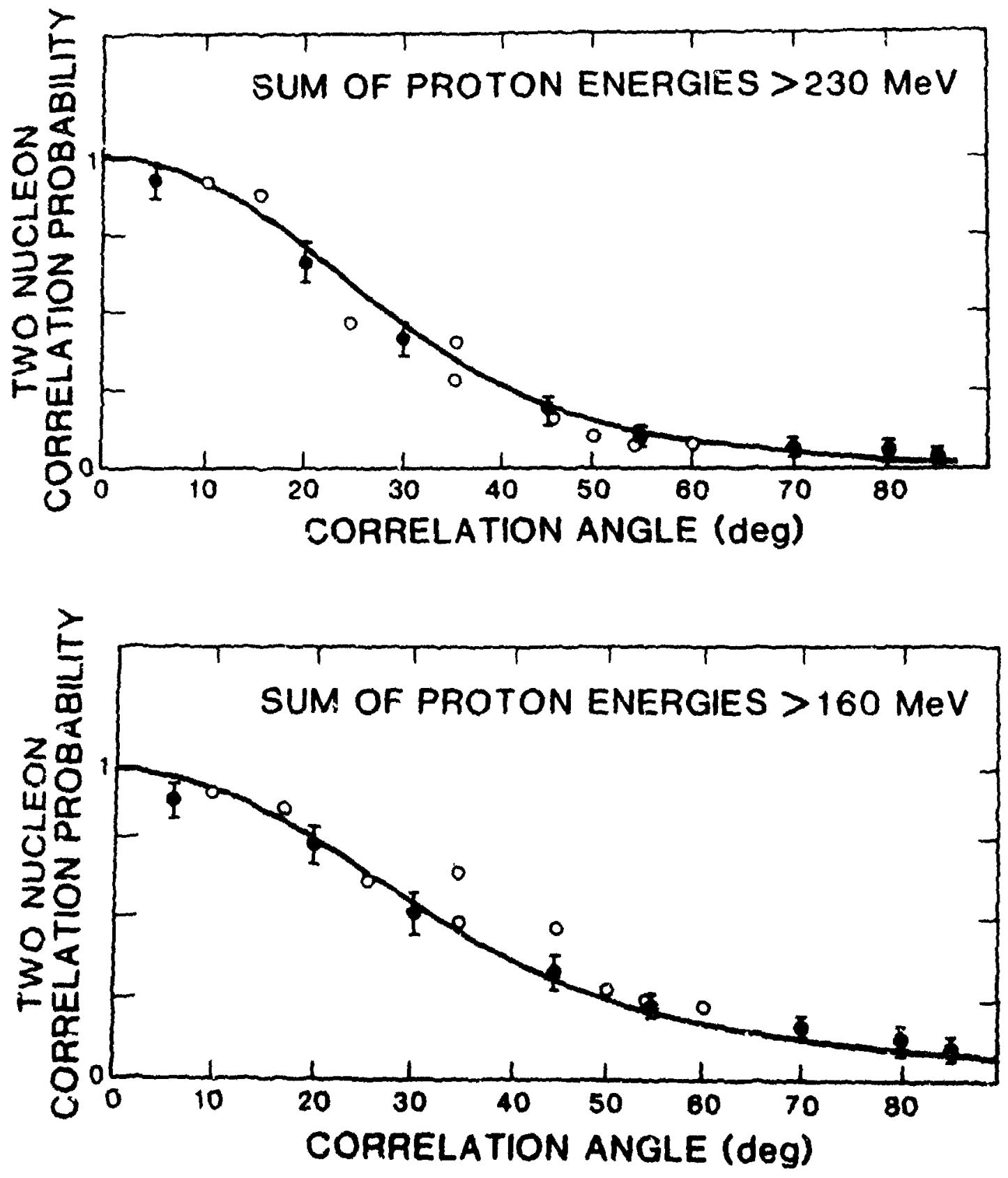

Figure. Predfcted angular correlation function coupared with the data . The incident pion momentum is $270 \mathrm{MeV} / \mathrm{c}$. The calculation avercges over 11 angles of alngle proton and out., as well su, in-plane events. The data corresponds to only in-plane events and the first proton counter fixud at $30^{\circ}$ (solld points) and $75^{\circ}$ (open clreles). The two polnts at $35^{\circ}$ cortesfond to measurements to the left and right of the two-body kinomatle polnt. 